\title{
25 Research Soure \\ Risk Factors For COVID-19 Positivity in Hospitalized Patients in A Low Prevalence Setting
}

\section{Iris Zohar}

Tel Aviv University

Orna Schwartz

Wolfson Medical Center

Debby Ben David

Tel Aviv University

\section{Margarita Mashavi}

Tel Aviv University

Mohamad Aboulil

Wolfson Medical Center

Orit Yossepowitch

Wolfson Medical Center

Shirley Shapiro Ben David

Wolfson Medical Center

Yasmin Maor ( $\nabla$ yasmin.maor@gmail.com )

Wolfson Medical Center https://orcid.org/0000-0001-7258-3434

\section{Original research}

Keywords: respiratory symptoms, LDH, LDH, COVID-19, emergency room

Posted Date: September 15th, 2020

DOI: https://doi.org/10.21203/rs.3.rs-72761/v1

License: (c) (i) This work is licensed under a Creative Commons Attribution 4.0 International License.

Read Full License 


\section{Abstract}

Background: Identifying hospitalized patients with Coronavirus disease 2019 (COVID-19) in a low prevalence setting is challenging. We aimed to identify differences between COVID-19 positive and negative patients.

Methods: Hospitalized patients with respiratory illness, or fever, were isolated in the emergency room and tested for COVID-19. Patients with a negative PCR and low probability for COVID-19 were taken out of isolation. Patients with a higher probability for COVID-19 remained in isolation during hospitalization and were retested after 48 hours. Risk factors for COVID-19 were assessed using logistic regression.

Results: 254 patients were included, 37 COVID-19-positive (14.6\%) and 217 COVID-19-negative (85.4\%). Median age was 76 years, $52 \%$ were males. In a multivariate regression model, variables significantly associated with COVID-19 positivity were exposure to a confirmed COVID-19 case, length of symptoms before testing, bilateral and peripheral infiltrates in chest X-ray, neutrophil count within the normal range, and elevated LDH. In an analysis including only patients with pneumonia $(\mathrm{N}=78,18$ positive for COVID19), only bilateral and peripheral infiltrates, normal neutrophil count and elevated LDH were associated with COVID-19 positivity.

Conclusions: The clinical presentation of COVID-19 positive and negative patients is similar, but radiographic and laboratory features may help to identify COVID-19 positive patients and to initiate quick decisions regarding isolation.

\section{Introduction}

In December 2019, a novel coronavirus, severe acute respiratory syndrome related to coronavirus 2 (SARS-CoV-2), emerged in Wuhan, Hubei, China, causing severe pneumonia [1]. Since then, corona virus disease 2019 (COVID-19) became prevalent worldwide and in 11th March 2020, the World Health Organization declared a pandemic caused by this virus. As of May $30^{\text {th, }} 2020$, more than 5.9 million people were infected worldwide, and more than 360,000 people have died. In Israel, 17,000 patients were diagnosed with COVID-19, and 284 patients died.

COVID-19 has a wide range of severity, and most patients have a mild illness and a good prognosis some require hospitalization. Usually at the beginning of the illness patients present with symptoms compatible with a flu like illness or an upper respiratory tract infection (URTI) and as disease progresses after several days about $20 \%$ of patients will develop frank pneumonia. Few will have additional complications including acute respiratory syndrome (ARDS), high levels of cytokines such as Interleukin-6 (IL-6), thromboembolic events, and multiorgan failure. The overall reported mortality is in the range of $1-10 \%$ [2-4].

The clinical presentation of COVID-19 is not specific and can mimic other viral infections. Symptoms such as fever, cough, malaise, and frank pneumonia are common for most viral respiratory tract 
infections including Influenza, Respiratory Syncytial Virus (RSV), Human Metapneumovirus (HMP) and many others [5]. This poses challenges to correct identification of COVID-19 patients. Laboratory diagnosis of SARS-CoV-2 is performed by real-time polymerase chain reaction (RT-PCR) on nasopharyngeal samples. Diagnosis is complicated by false-negative results that may reach $30 \%$ and false-positive results in the range of $1-2 \%$ [6-8].

COVID-19 is a highly contagious disease. Transmission to other persons occurs primarily by respiratory secretions through droplets, although other modes of transmission have been described. These include aerosol formations in patients on mechanical ventilation and transmission by contact with contaminated surfaces [9-10]. Isolation of patients with COVID-19 and treating these patients with appropriate personal protection equipment is important for reducing COVID-19 transmission.

Nonspecific clinical presentation and difficulties in interpreting PCR results pose challenges in differentiating between patients with and without COVID-19 for prompt isolation. This is particularly difficult in areas where the incidence of COVID-19 is low to moderate, as is the current situation in Israel. In places with low background transmission most patients with respiratory symptoms attending emergency rooms and patients hospitalized due to respiratory illness have other diagnoses. To date, there is limited data regarding the differences between hospitalized patients with confirmed COVID-19 and those who eventually were considered not to have COVID19 [11-12].

Wolfson Medical Center is a 700-bed hospital located in central Israel. During the first wave of the corona pandemic in Israel our hospital catered for approximately 100 COVID-19 patients. Many of these patients were referred for hospitalization after they were diagnosed in the community. In this study, we investigated the epidemiologic, clinical, radiographic and laboratory features of confirmed COVID-19 cases diagnosed upon admission to Wolfson Medical Center, or during their hospitalization, and compared them to hospitalized patients that were suspected of having COVID-19 but eventually were considered to have other diagnoses.

\section{Methods}

\section{Patients}

The study was performed in Wolfson Medical Center and approved by the hospitals Internal Review Board. We included all adult patients, who were hospitalized between March $15^{\text {th }} 2020$ and May $15^{\text {th }}$ 2020, and had confirmed COVID-19 diagnosed at admission or during hospitalization, and a convenient sample of patients who were suspected to have COVID-19 but this diagnosis was ruled out. In some cases, the question of COVID-19 infection was raised only several days after admission. For these patients, the data was collected at the day the first SARS-CoV-2 test was performed (index date). Suspected cases were tested for SARS-CoV-2 by RT-PCR at presentation. If the result was negative, cases were reviewed by a physician from the Infectious Diseases Unit to determine the probability of COVID-19 infection. Cases that were defined as high probability were tested again after 48 hours and remained in 
isolation. In cases that were defined as low probability isolation measures were stopped. Criteria for remaining in isolation were peripheral lung infiltrates on chest X-ray or chest cat-scan, fever with no alternative explanation, unexplained hypoxemia, and lymphopenia below 1.0 cellx $10^{3} /$ liter.

Patients who were diagnosed with COVID-19 before admission to the hospital were excluded.

\section{Diagnosis of COVID-19}

Diagnosis of COVID-19 was done by detection of SARS-CoV-2 RNA by RT-PCR obtained from the nasopharynx or in patients on mechanical ventilation by a sample obtained from deep suction.

Kits for RT-PCR varied according to availability. All tests were approved by the Israeli Ministry of Health. We used the following kits: Seegene, GeneExpert and BGI. Tests were performed and interpreted according to the manufacturer's instructions. Results were positive, negative, or inconclusive. In all inconclusive cases a second test was performed, and the final interpretation was based on the pretest probability in discussion with physicians from the Infectious Disease Unit.

\section{Data collection}

We reviewed patients' clinical charts, laboratory exams and imaging results on the day in which the first COVID-19 test was performed, at admission, or during hospitalization prior to receiving the results of the PCR tests. Patients' data was recorded in a tabular manner. Information included epidemiologic data, clinical presentation, laboratory results and radiological characteristics. Uncertainties were discussed with the attending physician. For each patient we determined if the clinical presentation was pneumonia, upper respiratory tract infection (URTI), other fever syndromes or isolated dyspnea.

URTI was defined as having respiratory symptoms, fever, and on chest X-ray no new lung infiltrates compatible with an infectious process.

Pneumonia was defined as having respiratory symptoms, fever, and on chest X-ray new lung infiltrates compatible with an infectious process.

\section{Statistical analysis}

Statistical analyses were performed using IBM SPSS ${ }^{\circledR}$ version 25. Categorical variables were presented as absolute number and percent, and continuous variables were presented as median and intra-quartile range (IQR). Confirmed and non-confirmed COVID-19 patients were compared using Pearson Chi-Square test and Fisher Exact test for categorical variables and Student T-test and Mann-Whitney test for continuous variables. We assessed risk factors for confirmed COVID-19 using logistic regression. Independent variables found to be significantly associated with the dependent variable in a univariate analysis $(p<0.1)$ were entered into multivariate binary logistic regression analysis, (backwards conditional), with results presented as odds ratio (OR) with a confidence interval (Cl) of 95\%. Statistical significance was set at $p<0.05$. 
Since we assumed the clinical factors might differ between patients with or without pneumonia, we performed another logistic regression for the subgroup of patients with pneumonia, excluding other diagnoses. We also performed a logistic regression analysis including only non-COVID-19 cases with two negative tests (patients with a higher pretest probability for COVID-19).

\section{Results}

The study included 254 patients, 37 COVID-19-positive (14.6\%) and 217 COVID19-negative (85.4\%) patients (Fig. 1). Thirty-five (94.6\%) of the positive patients were tested positive on the index date (the date the first RT-PCR was performed). Two patients (5.4\%) tested negative on the index date and positive in the following test performed 48 hours later. Of the 217 COVID-19-negative patients, 107 (49.3\%) were considered to have a low probability for COVID-19 infection and were tested once. One hundred and ten patients $(50.7 \%)$ had a higher probability of having COVID-19 and thus were tested twice. None of the patients that were taken out of isolation were later found to have COVID-19 infection, so our algorithm proved to be safe.

The median age was 76 years, $52 \%$ were male, and $28 \%$ were debilitated and suffered from dementia (Table 1). Two hundred and thirty three patients (91.7\%) were suspected to have COVID-19 at admission with the first test performed in the emergency department, while for the remaining 21 (8.3\%) patients suspicion for COVID-19 was raised 72 hours or more after admission to the hospital. Of these, 13 were hospitalized in the internal medicine departments, four in general surgery, and four in the orthopedics department. 
Table 1

Patients' characteristics.

\begin{tabular}{|c|c|c|c|c|c|c|}
\hline & \multicolumn{2}{|c|}{$\begin{array}{l}\text { All Patients } \\
\mathrm{N}=254\end{array}$} & & \multicolumn{3}{|c|}{$\begin{array}{l}\text { Pneumonia } \\
\mathrm{N}=78\end{array}$} \\
\hline & $\begin{array}{l}\text { COVID-19 } \\
\text { positive } \\
\text { patients } \\
\mathrm{N}=37 \\
(14.6 \%)\end{array}$ & $\begin{array}{l}\text { COVID-19 } \\
\text { negative } \\
\text { patients } \\
\mathrm{N}=217 \\
(85.4 \%)\end{array}$ & $\begin{array}{l}P \\
\text { value }\end{array}$ & $\begin{array}{l}\text { COVID- } \\
19 \\
\text { positive } \\
\text { patients } \\
\begin{array}{l}\mathrm{N}=18 \\
(23.1 \%)\end{array}\end{array}$ & $\begin{array}{l}\text { COVID- } \\
19 \\
\text { negative } \\
\text { patients } \\
\mathrm{N}=60 \\
(76.9 \%)\end{array}$ & $\begin{array}{l}P \\
\text { value }\end{array}$ \\
\hline Age - years, median (IQR) & $\begin{array}{l}76 \\
(64.5- \\
84.0)\end{array}$ & $\begin{array}{l}76 \\
(63.5- \\
85.5)\end{array}$ & 0.967 & $\begin{array}{l}78.0 \\
(65.3- \\
88.3)\end{array}$ & $\begin{array}{l}79.5 \\
(64.5- \\
89.0)\end{array}$ & 0.887 \\
\hline Sex - male n (\%) & $23(62.2)$ & $109(50.2)$ & 0.214 & $\begin{array}{l}13 \\
(72.2)\end{array}$ & $29(48.3)$ & 0.106 \\
\hline $\begin{array}{l}\text { Hospitalization in the last } 30 \\
\text { days in a hospital or a long- } \\
\text { term care facility n (\%) }\end{array}$ & $19(51.4)$ & $82(37.8)$ & 0.146 & $9(50.0)$ & $31(51.7)$ & 1 \\
\hline Debilitated with dementia n (\%) & $7(18.9)$ & $64(29.5)$ & 0.236 & $3(16.7)$ & $26(43.3)$ & 0.53 \\
\hline Health care worker n (\%) & $3(8.1)$ & $1(0.5)$ & 0.010 & $1(5.6)$ & 0 & 0.231 \\
\hline $\begin{array}{l}\text { Exposure to a known COVID-19 } \\
\text { case } \mathrm{n}(\%)\end{array}$ & $13(35.1)$ & $3(1.4)$ & $<.001$ & $2(11.1)$ & $3(5.0)$ & 0.326 \\
\hline Clinical presentation & & & 0.048 & & & 1 \\
\hline - URTI n (\%) & $7(18.9)$ & $35(16.1)$ & & & & \\
\hline - Pneumonia n (\%) & $18(48.6)$ & $60(27.6)$ & & $18(100)$ & $60(100)$ & \\
\hline - Fever n (\%) * & $6(16.2 \%)$ & $45(20.7)$ & & & & \\
\hline - Dyspnea without fever $\mathrm{n}(\%)$ & $4(10.8)$ & $67(30.9)$ & & & & \\
\hline - Nonspecific symptoms n (\%) & $2(5.4)$ & $10(4.6)$ & & & & \\
\hline
\end{tabular}

$\mathrm{L}$ - liter; ALT - alanine transaminase; AST - aspartate aminotransferase; LDH - lactate dehydrogenase

* Fever without pneumonia or URTI

** For the number of symptomatic days prior to presentation we had data for 195 patients. For X-ray findings we had data for 252 patients. For ALT we had data for 232 patients, for AST we had data for 231 patients and for LDH we had data for 203 patients 


\begin{tabular}{|c|c|c|c|c|c|c|}
\hline \multirow{4}{*}{$\begin{array}{l}\text { Length of symptoms before } \\
\text { the index date (days)median } \\
\text { (IQR) } \star \star\end{array}$} & \multirow{2}{*}{\multicolumn{2}{|c|}{$\begin{array}{l}\text { All Patients } \\
\mathrm{N}=254\end{array}$}} & & \multirow{2}{*}{\multicolumn{3}{|c|}{$\begin{array}{l}\text { Pneumonia } \\
N=78\end{array}$}} \\
\hline & & & & & & \\
\hline & 5.0 & 2.0 & \multirow{2}{*}{$<001$} & 6.5 & 2.0 & \multirow[t]{2}{*}{0.003} \\
\hline & $(2.0-7.0)$ & $(1.0-3.0)$ & & $\begin{array}{l}(2.0- \\
10.0)\end{array}$ & $\begin{array}{l}(1.0- \\
3.0)\end{array}$ & \\
\hline Mechanical ventilation n (\%) & $3(8.1)$ & $27(12.4)$ & 0.588 & $3(16.7)$ & $6(10.0)$ & 0.423 \\
\hline $\mathrm{O}_{2}$ therapy $\mathrm{n}(\%)$ & $15(40.5)$ & $115(43.0)$ & 0.213 & $9(50.0)$ & $36(58.3)$ & 0.594 \\
\hline Fever $>38^{\circ} \mathrm{C} n(\%)$ & $23(62.2)$ & $118(54.4)$ & 0.475 & $\begin{array}{l}11 \\
(61.1)\end{array}$ & $42(70.0)$ & 0.567 \\
\hline Cough n (\%) & $14(35.1)$ & $65(30.0)$ & 0.565 & $7(38.9)$ & $20(33.3)$ & 0.779 \\
\hline Dyspnea n (\%) & $15(40.5)$ & $123(56.7)$ & 0.076 & $\begin{array}{l}12 \\
(66.7)\end{array}$ & $33(55.0)$ & 0.427 \\
\hline Diarrhea n (\%) & $4(10.8)$ & $8(3.7)$ & 0.080 & $2(11.1)$ & 0 & 0.051 \\
\hline \multicolumn{7}{|l|}{ Chest X-ray findings ** } \\
\hline - Any infiltrates n (\%) & $23(63.9)$ & $79(36.6)$ & 0.003 & $18(100)$ & $60(100)$ & 1 \\
\hline - bilateral infiltrates n (\%) & $14(38.9)$ & $22(10.2)$ & $<.001$ & $\begin{array}{l}10 \\
(55.6)\end{array}$ & $14(23.3)$ & 0.018 \\
\hline - peripheral infiltrates n (\%) & $21(58.3)$ & $35(16.2)$ & $<.001$ & $\begin{array}{l}16 \\
(88.9)\end{array}$ & $26(43.3)$ & 0.001 \\
\hline WBC - median (IQR) $\left(\times 10^{3} / \mathrm{L}\right)$ & $\begin{array}{l}6.7(4.2- \\
11.3)\end{array}$ & $\begin{array}{l}10.7(7.4- \\
14.3)\end{array}$ & 0.001 & $\begin{array}{l}8.3(6.0- \\
13.1)\end{array}$ & $\begin{array}{l}11.5 \\
(8.1- \\
15.0)\end{array}$ & 0.87 \\
\hline $\begin{array}{l}\text { Lymphocytes }-\left(x 10^{3} / L\right) \text { median } \\
\text { (IQR) }\end{array}$ & $\begin{array}{l}0.9(0.6- \\
1.3)\end{array}$ & $\begin{array}{l}1.2(0.7- \\
1.9)\end{array}$ & 0.011 & $\begin{array}{l}0.8 \\
(0.6- \\
1.3)\end{array}$ & $\begin{array}{l}1.0(7.0- \\
1.5)\end{array}$ & 0.172 \\
\hline - Lymphocytes $<1 \times 10^{3} / \mathrm{L}$ & $22(59.5)$ & $82(37.8)$ & 0.018 & $\begin{array}{l}12 \\
(66.7)\end{array}$ & $24(40.0)$ & 0.061 \\
\hline $\begin{array}{l}\text { Neutrophils - }\left(x 10^{3} / \mathrm{L}\right) \text { median } \\
(\mathrm{IQR})\end{array}$ & $\begin{array}{l}4.8(3.0- \\
7.6)\end{array}$ & $\begin{array}{l}8.4(5.2- \\
11.4)\end{array}$ & $\begin{array}{l}< \\
0.001\end{array}$ & $\begin{array}{l}5.5 \\
(3.6- \\
9.3)\end{array}$ & $\begin{array}{l}9.3(6.2- \\
12.2)\end{array}$ & 0.006 \\
\hline
\end{tabular}

L - liter; ALT - alanine transaminase; AST - aspartate aminotransferase; LDH - lactate dehydrogenase

* Fever without pneumonia or URTI

** For the number of symptomatic days prior to presentation we had data for 195 patients. For X-ray findings we had data for 252 patients. For ALT we had data for 232 patients, for AST we had data for 231 patients and for LDH we had data for 203 patients 


\begin{tabular}{|c|c|c|c|c|c|c|}
\hline \multirow{3}{*}{$\begin{array}{l}- \text { Neutrophils }>7.5 \times 10^{3} / \mathrm{L} \mathrm{n} \\
(\%)\end{array}$} & \multirow{2}{*}{\multicolumn{2}{|c|}{$\begin{array}{l}\text { All Patients } \\
\mathrm{N}=254\end{array}$}} & & \multirow{2}{*}{\multicolumn{3}{|c|}{$\begin{array}{l}\text { Pneumonia } \\
\mathrm{N}=78\end{array}$}} \\
\hline & & & & & & \\
\hline & $10(27.0)$ & $122(56.2)$ & 0.001 & 7 (38.9) & $41(68.3)$ & 0.030 \\
\hline $\mathrm{ALT}>40 \mathrm{U} / \mathrm{L}^{\star \star}$ & $5(15.2)$ & $38(19.1)$ & 0.809 & $2(11.1)$ & $12(21.4)$ & 0.495 \\
\hline $\mathrm{AST}>40 \mathrm{U} / \mathrm{L}^{\star *}$ & $6(18.8)$ & $43(21.6)$ & 0.819 & $5(27.8)$ & $12(21.4)$ & 0.748 \\
\hline LDH U/L median (IQR) ** & $\begin{array}{l}608 \\
(443- \\
687)\end{array}$ & $\begin{array}{l}413(343- \\
570)\end{array}$ & 0.006 & $\begin{array}{l}619 \\
(518- \\
768)\end{array}$ & $\begin{array}{l}427 \\
(374- \\
562)\end{array}$ & 0.004 \\
\hline - LDH > $500 \mathrm{U} / \mathrm{L}$ & $17(65.4)$ & $56(31.6)$ & 0.002 & $\begin{array}{l}12 \\
(80.0)\end{array}$ & $16(33.3)$ & 0.002 \\
\hline $\begin{array}{l}\text { CRP (mg/deciliter)- median } \\
\text { (IQR) }\end{array}$ & $\begin{array}{l}8.97 \\
(2.49- \\
15.96)\end{array}$ & $\begin{array}{l}4.99 \\
(1.73- \\
12.54)\end{array}$ & 0.201 & $\begin{array}{l}9.61 \\
(7.78- \\
20.04)\end{array}$ & $\begin{array}{l}8.45 \\
(3.64- \\
17.69)\end{array}$ & 0.305 \\
\hline \multicolumn{7}{|c|}{$\begin{array}{l}\mathrm{L} \text { - liter; ALT - alanine transaminase; AST - aspartate aminotransferase; LDH - lactate } \\
\text { dehydrogenase }\end{array}$} \\
\hline \multicolumn{7}{|c|}{ * Fever without pneumonia or URTI } \\
\hline \multicolumn{7}{|c|}{$\begin{array}{l}\text { ** For the number of symptomatic days prior to presentation we had data for } 195 \text { patients. For X-ray } \\
\text { findings we had data for } 252 \text { patients. For ALT we had data for } 232 \text { patients, for AST we had data for } \\
231 \text { patients and for LDH we had data for } 203 \text { patients }\end{array}$} \\
\hline
\end{tabular}

Clinical presentation was diverse. In COVID-19 positive patients the most common findings were pneumonia [18 patients, (48.6\%)] and URTI [seven patients, (18.9\%)], while in COVID-19 negative patients dyspnea without fever [67 patients, (30.9\%)], pneumonia [60 patients, (27.6\%)] and fever without a defined respiratory syndrome [45 patients, $(20.7 \%)$ ] were the most common presentation. Information regarding length of symptoms before presentation was available in 195 patients (76.8\%) - COVID-19 positive patients presented later in the course of their illness as compared to COVID-19 negative patients (5 versus 2 days, $p<0.001$ ) (Fig. 2).

Pulmonary infiltrates were more common in COVID-19 positive patients, and patients with infiltrates had a higher rate of bilateral infiltrates and of peripheral infiltrates (Table 1). COVID-19-positive patients had a higher rate of leukopenia and lymphopenia and lower rate of neutrophilia. They also had higher rates of elevated lactate dehydrogenase (LDH) (> $500 \mathrm{U} / \mathrm{L})$, although LDH elevation in most cases was moderate (in the range of 500-1,000 U/L). Liver enzymes were elevated in a minority of patients in both groups, and there was no difference in C-reactive protein (CRP) levels.

In a multivariate analysis (Table 2), variables significantly associated with COVID-19 positivity included exposure to a known COVID-19 case, longer duration of symptoms before COVID-19 testing, bilateral 
pulmonary infiltrates, peripheral pulmonary infiltrates, an absolute neutrophil count lower than $7.5 \times 10^{3} / \mathrm{L}$ and LDH levels $>500 \mathrm{U} / \mathrm{L}$.

Table 2

Multivariate logistic regression $(n=155)$ assessing risk factors for COVID-19 positivity.

\begin{tabular}{|llll|}
\hline & Univariate & Multivariate & \\
\hline Age & P & OR $(95 \%$ Cl $)$ & P \\
\hline Sex & 0.967 & & \\
\hline Health care worker & 0.214 & & \\
\hline Recent exposure to a COVID-19 patient & 0.010 & & \\
\hline Length of symptoms before the index date (days) & $<0.001$ & $1.15(1-1.32)$ & 0.055 \\
\hline Bilateral lung infiltrates & $<0.001$ & $91.37(9.49-879.57)$ & $<0.001$ \\
\hline Peripheral lung infiltrates & $<0.001$ & $5.11(1.34-19.53)$ & 0.017 \\
\hline Neutrophil count $<7.5 \times 10^{3} / \mathrm{L}$ & 0.001 & $3.35(0.83-13.63)$ & 0.091 \\
\hline LDH $>500$ U $/ \mathrm{L}$ & 0.006 & $6.82(1.55-29.94)$ & 0.011 \\
\hline$* \mathrm{R}^{2}$ by Cox and Snell -0.310 & & & \\
\hline
\end{tabular}

In a similar analysis, including all positive patients and only the COVID-19 negative patients considered by the Infectious Disease Specialists to have a high pretest probability for COVID-19 infection, and thus had an additional negative test 48 hours after the index date $(n=110)$ results were similar.

Next, we performed another analysis including only patients with pneumonia ( $N=78,18$ COVID-19positive patients and 60 COVID-19-negative patients). Median age was 78 years, and $53.8 \%$ were male (Table 1). A third of pneumonias patients were identified 72 hours or more after admission (similarly in both groups). Clinical features were mostly similar between COVID-19 positive and negative patients, but diarrhea was present in two COVID-19-positive patients and in none of the COVID-19-negative patients ( $P$ $=0.051$ ). Bilateral and peripheral infiltrates were more common in the COVID-19-positive group. COVID-19positive patients with pneumonia had a higher rate of leukopenia, lower rate of neutrophilia and a higher rate of elevated LDH. Multivariate analysis (Table 3) showed that bilateral infiltrates, peripheral infiltrates, lack of absolute neutrophilia and elevated LDH were associated with COVID-19 positivity. 
Table 3

Multivariate logistic regression $(n=50)$ assessing risk factors for COVID-19 positivity in patients with pneumonia.

\begin{tabular}{|llll|}
\hline & Univariate & Multivariate & \\
\hline Age & $\mathrm{P}$ & OR $(95 \% \mathrm{Cl})$ & $\mathrm{P}$ \\
\hline Sex & 0.887 & & \\
\hline Health care worker & 0.106 & & \\
\hline Recent exposure to a COVID-19 patient & 0.231 & & \\
\hline Length of symptoms before COVID-19 testing (days) & 0.003 & & 0.019 \\
\hline Bilateral lung infiltrates & 0.326 & & 0.036 \\
\hline Peripheral lung infiltrates & 0.018 & $10.43(1.46-74.43)$ & 0.084 \\
\hline Neutrophile count $<x 10^{3} / \mathrm{L}$ & 0.001 & $5.14(0.80-32.92)$ & 0.018 \\
\hline LDH $>500$ U/L & 0.030 & $11.53(1.51-87.91)$ & 0 \\
\hline * $\mathrm{R}^{2}$ by Cox and Snell -0.402 & 0.002 & & \\
\hline
\end{tabular}

\section{Discussion}

In this study, we aimed to find characteristics that may differentiate between patients with or without PCR confirmed COVID-19, in a population of hospitalized patients with suspected COVID-19. We found several parameters in the laboratory and radiographic results which are significantly more common among COVID-19 positive patients, including peripheral and bilateral infiltrates in chest X-ray, elevated LDH, and lack of absolute neutrophilia.

Only two studies [11-12] assessed the ability to differentiate between COVID-19 positive and negative patients in a hospital setting but they were performed in a high-incidence setting area (Wuhan, China) at the beginning of the pandemic, and thus included only a small number of COVID-19-negative patients. Our study was performed in a low-incidence setting, thus having a different perspective on the pre-test probability of a patient having COVID-19. Also, both studies included only patients admitted with pneumonia, while our study included more diverse presentations, reflecting multiple stages in the natural history of COVID-19.

In a low COVID-19 incidence setting, one of the main challenges is identifying and isolating promptly COVID-19 positive patients to prevent hospital outbreaks. Because of the relatively low sensitivity of PCR tests, it is important to identify patients with high probability for COVID-19, and to continue isolation, until repeated testing is performed. Because in a low setting the number of COVID-19 negative patients is high, 
this poses a significant challenge in triaging patients with low and high probability for COVID-19 infection. Identifying features specific for COVID-19-positive patients may help in performing this task.

The parameters assessed in our study can be divided to four groups, epidemiological, clinical, radiographic, and laboratory tests. Of the epidemiological parameters, working in the health care system was associated with COVID-19 positivity as shown previously [13]. Unsurprisingly, exposure to a confirmed case was also associated with COVID-19 infection.

Clinical symptoms were similar between COVID-19 positive and negative patients, as was shown in previous studies [11-12]. Clinical syndromes were different, with COVID-19 positive patients presenting mostly with pneumonia or URTI, and COVID-19 negative patients having more diverse and less specific presentations, reflecting a high index of suspicion for COVID-19. We also showed that COVID-19 positive patients presented after a longer period of symptoms than COVID-19 negative patients. This is compatible with previous studies showing a mild disease in the first week of COVID-19, not justifying hospitalization [3-4].

Presence of infiltrates in chest X-ray, and specifically bilateral and peripheral infiltrates, were more common in COVID-19 patients, in accordance with the known radiographic features of COVID-19 [14]. It has been previously described that elevated LDH levels together with elevated liver enzymes can differentiate between COVID-19 positive and negative patients [11]. In our cohort only elevated LDH differed between COVID-19 positive and negative patients, and there was no difference in liver enzyme levels between the two groups.

Our approach proved to be safe. In none of the cases COVID-19 was identified after patients were taken out of isolation.

There are several limitations to our study. First, in some patients not all the laboratory data was available. Second, since in our hospital during the study period there was no strict definition for a suspected COVID19 patient, the COVID-19 negative group is heterogenous and includes patients with atypical presentation with a range of pretest probability for COVID-19. This reflects the real-life situation that health care personnel need to face with during this pandemic. We dealt with these issues in several ways. We did an additional analysis including only patients with a high pre-test probability for COVID-19. This yielded similar results. We also did another analysis that included only patients presenting with pneumonia. This again yielded similar results, but with less power due to the smaller sample included. Patients included in this study presented in different stages of COVID-19. The evolving nature of COVID-19 which starts as a viral URTI, progresses to pneumonia in various degrees of severity, and only later to multiorgan failure, cytokine storm, and need for mechanical ventilation makes it difficult to discuss specific clinical features, as they are related to the stage of COVID-19. Another limitation is that this was a single-center study, performed at a secondary medical center. Thus, we did not have many immunocompromised patients, and we had a high proportion of elderly patients. This may limit the generalization of the results. Also, these results are relevant for a setup with a low to moderate incidence of COVID-19, where many patients 
hospitalized suffer from other medical problems. In a high incidence setting of COVID-19, decisions regarding rapid diagnosis and isolation may differ.

\section{Conclusions}

The clinical presentation of COVID-19 positive and negative patients is similar, but several radiographic and laboratory features can help to identify COVID-19 positive patients: bilateral and peripheral infiltrates on chest X-ray, a neutrophil count within normal limits, and elevated LDH. Identifying these features can help to initiate quick decisions regarding isolation of these patients.

\section{Declarations}

The study was approved by the hospitals internal review committee (0156-20-WOMC). We received a waiver of informed consent from the committee.

All authors read the manuscript and approved its publication.

Data is available upon request.

All other authors have nothing to declare and no competing interests.

The study was performed by use of internal funds of the Infectious Disease Unit, Wolfson Medical Center.

\section{Author Contributions}

IZ - conceptualization, data curation, writing

OS- laboratory analysis

DBD - review and editing

MM - data curation, review and editing

MAl - data curation

OY - review and editing

SSBD - review and editing

YM - conceptualization, statistical analysis, review and editing, funding

\section{References}

1. Zhu N, Zhang D, Wang W, et al. A Novel Coronavirus from Patients with Pneumonia in China, 2019. NEJM. 2020;382:727-33. 
2. Chen N, Zhou M, Dong X, et al. Epidemiological and clinical characteristics of 99 cases of 2019 novel coronavirus pneumonia in Wuhan, China: a descriptive study. Lancet. 2020;395:507-13.

3. Huang C, Wang Y, Li X, et al. Clinical features of patients infected with 2019 novel coronavirus in Wuhan, China. Lancet. 2020;395:497-506.

4. Zhou F, Yu T, Du R, et al. Clinical course and risk factors for mortality of adult inpatients with COVID19 in Wuhan, China: a retrospective cohort study. Lancet. 2020;395:1054-62.

5. Marcos MA, Esperatti M, Torres A. Viral Pneumonia. Curr Opin Infect Dis. 2009;22:143-7.

6. Wang W, Xu Y, Gao R, et al. Detection of SARS-CoV-2 in Different Types of Clinical Specimens. JAMA. 2020;323:1843-4.

7. Tahamtan A, Ardebili A. Real-time RT-PCR in COVID-19 detection: issues affecting the results. Expert Rev Mol Diagn. 2020;20:453-4.

8. Kim H, Hong H, Yoon SH. Diagnostic Performance of CT and Reverse Transcriptase-Polymerase Chain Reaction for Coronavirus Disease 2019: A Meta-Analysis. Radiology. 2020. doi:10.1148/radiol.2020201343 [Online ahead of print].

9. Guo ZD, Wang ZY, Zhang SF, et al. Aerosol and Surface Distribution of Severe Acute Respiratory Syndrome Coronavirus 2 in Hospital Wards, Wuhan, China, 2020. Emerg Infect Dis. 2020;26(7).

10. World health organization. Modes of transmission of virus causing COVID-19: implications for IPC precaution recommendations [Internet]. World Health Organization; 2020. Available from: https://apps.who.int/iris/bitstream/handle/10665/331601/WHO-2019-nCoV-Sci_BriefTransmission_modes-2020.1-eng.pdf.

11. Zhao D, Yao F, Wang L, et al. A comparative study on the clinical features of COVID-19 pneumonia to other pneumonias. Clin Infect Dis. 2020. doi:10.1093/cid/ciaa247 [Online ahead of print].

12. Chen $X$, Yang $Y$, Huang $M$, et al. Differences Between COVID-19 and Suspected Then Confirmed SARS-CoV-2-negative Pneumonia: A Retrospective Study from a Single Center. J Med Virol. 2020. doi:10.1002/jmv.25810 [Online ahead of print].

13. Kluytmans-van den Bergh MFQ, Buiting AGM, Pas SD, et al. Prevalence and Clinical Presentation of Health Care Workers with Symptoms of Coronavirus Disease 2019 in 2 Dutch Hospitals During an Early Phase of the Pandemic. JAMA Netw Open. 2020;3(5):e209673.

14. Vancheri SG, Savietto G, Ballati F, et al. Radiographic findings in 240 patients with COVID-19 pneumonia: time-dependence after the onset of symptoms. Eur Radiol. 2020:19.doi:10.1007/s00330-020-06967-7 [Online ahead of print].

\section{Figures}




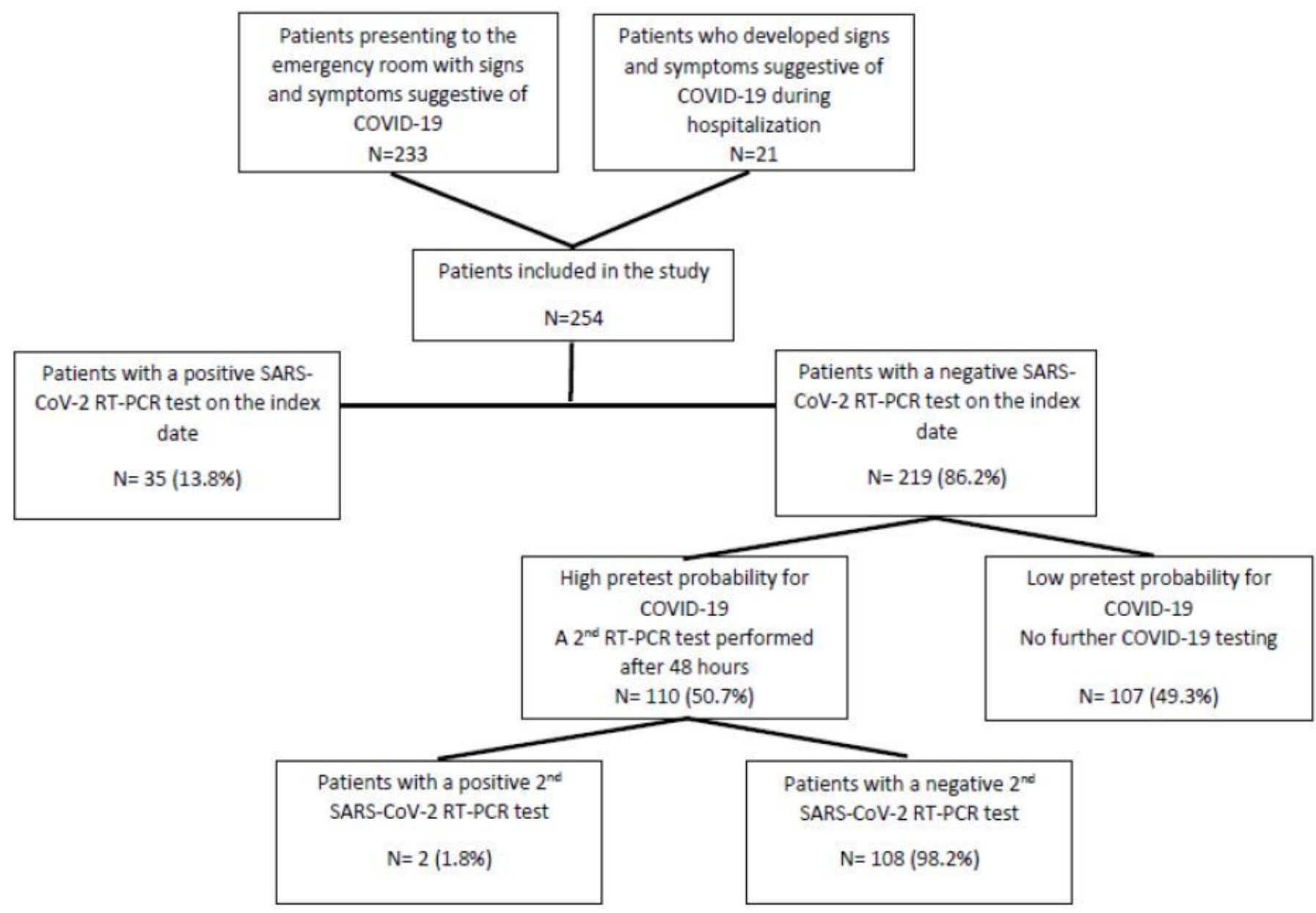

\section{Figure 1}

Description of patients included in the study. Patients that had signs and symptoms compatible with COVID-19 were tested at the emergency room. If patients developed signs and symptoms suggestive of COVID-19 during hospitalization they were tested during hospitalization. Patients with negative test results and a low pretest probability for COVID-19 after a review of a physician from the Infection Disease Unit were taken out of isolation. Patients with a higher pretest probability for COVID-19 were tested again after 48 hours. 


\section{Percent of patients}

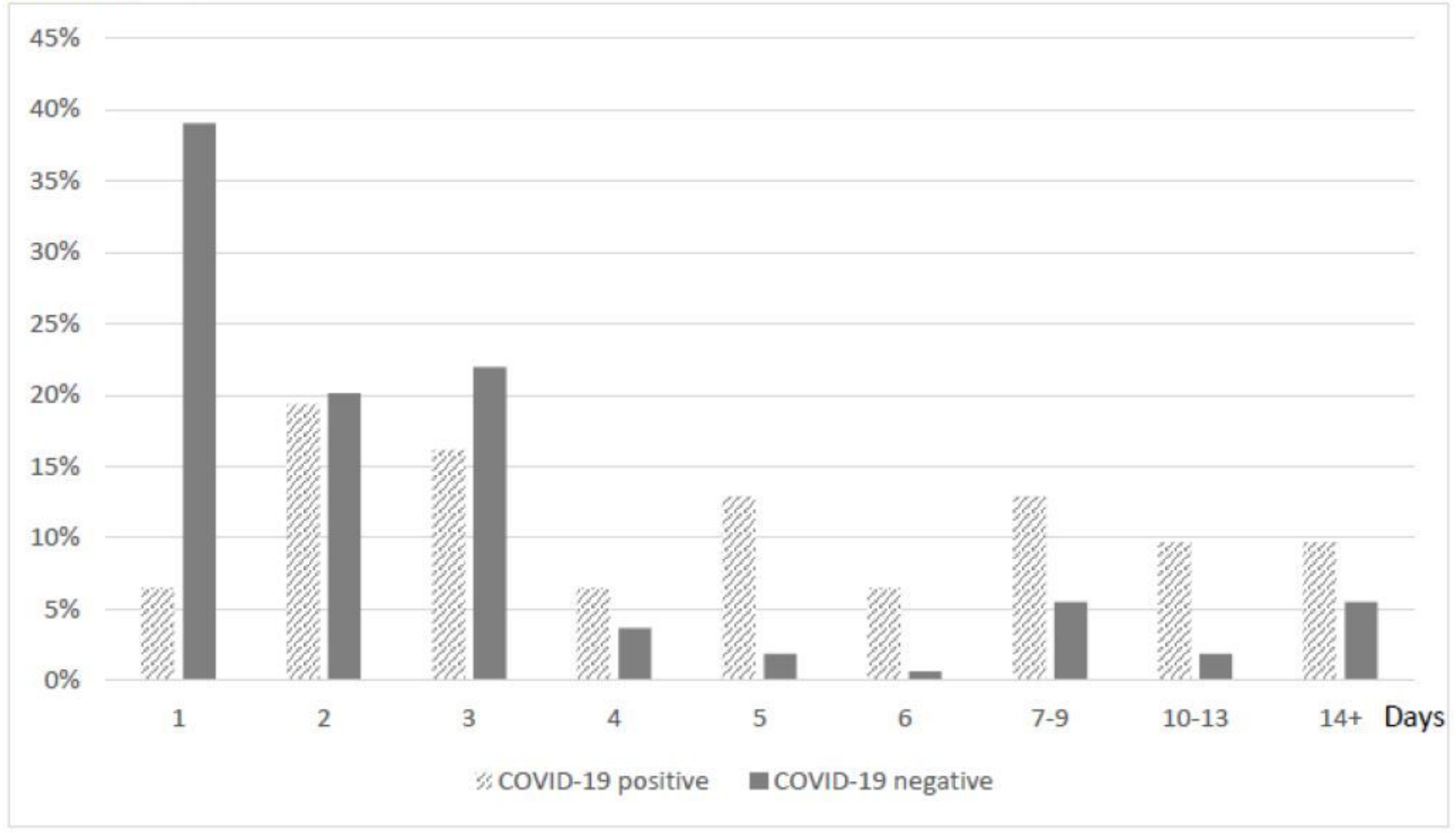

Figure 2

Distribution of duration of symptoms before performing the first test for COVID-19. Duration of symptoms before testing for COVID-19. COVID-19 negative patients [n=164, (84\%)] (dark grey) presented after 1 to 3 days after symptoms began, while COVID-19 positive patients [n=31 (16\%)] (textured grey) presented after 1 to 3 days after symptoms began. 